\title{
UNIFORM ASYMPTOTIC NORMAL STRUCTURE, THE UNIFORM SEMI-OPIAL PROPERTY, AND FIXED POINTS OF ASYMPTOTICALLY REGULAR UNIFORMLY LIPSCHITZIAN SEMIGROUPS. PART II
}

\author{
MONIKA BUDZYŃSKA, TADEUSZ KUCZUMOW AND SIMEON REICH
}

ABSTRACT. In this part of our paper we present several new theorems concerning the existence of common fixed points of asymptotically regular uniformly lipschitzian semigroups.

\section{INTRODUCTION}

Let $(X,\|\cdot\|)$ be a Banach space and $C$ a subset of $X$. A mapping $T$ : $C \rightarrow C$ is said to be uniformly $k$-lipschitzian if for each $x, y \in C$ and every natural number $n,\left\|T^{n} x-T^{n} y\right\| \leq k\|x-y\|$. If $k=1$, then the mapping $T$ is called nonexpansive. These definitions can also be introduced in metric spaces. The class of uniformly lipschitzian mappings on $C$ is completely characterized as the class of those mappings on $C$ which are nonexpansive with respect to some metric on $C$ which is equivalent to the norm [16]. In this part of our paper we use the new geometric coefficients introduced in its first part to study the existence of (common) fixed points for this class of mappings.

\section{BASiC NOTATIONS AND FACTS}

Throughout this paper we will use the notations from the first part of our paper [6]. However, before we recall several known fixed point theorems we need a few additional notations.

1991 Mathematics Subject Classification. 47H10, 47H20.

Key words and phrases. The uniform semi-Opial property, asymptotically regular and uniformly lipschitzian semigroups, fixed points.

Received: September 15, 1997. 
Let $(X,\|\cdot\|)$ denote a Banach space. For $x \in X$ and a bounded sequence $\left\{x_{n}\right\}$ the asymptotic radius of $\left\{x_{n}\right\}$ at $x$ is the number $r_{a}\left(x,\left\{x_{n}\right\}\right)$ $=\lim \sup _{n}\left\|x-x_{n}\right\|$. Now for a nonempty closed convex subset $C$ of $X$ the asymptotic radius of $\left\{x_{n}\right\}$ in $C$ is the number $r\left(C,\left\{x_{n}\right\}\right)=\inf \left\{r_{a}\left(x,\left\{x_{n}\right\}\right)\right.$ : $x \in C\}$. The asymptotic center of $\left\{x_{n}\right\}$ in $C[13]$ is the set $A c\left(C,\left\{x_{n}\right\}\right)=$ $\left\{x \in C: r_{a}\left(x,\left\{x_{n}\right\}\right)=r\left(C,\left\{x_{n}\right\}\right)\right\}$. For more details see [1], [16] and [17].

Let $\bar{B}(0,1)$ be the closed unit ball in $X$. The modulus of convexity of $X$ is the function $\delta:[0,2] \rightarrow[0,1]$ defined by

$$
\delta(\epsilon)=\inf \left\{1-\frac{\|x+y\|}{2} ; x, y \in \bar{B}(0,1),\|x-y\| \geq \epsilon\right\}
$$

[8]. The characteristic of convexity of $X$ is the number $\epsilon_{0}(X)=$ $\sup \{\epsilon: \delta(\epsilon)=0\}$ [16]. When $\epsilon_{0}(X)=0 X$ is called a uniformly convex space [8].

The Lifshitz characteristic $\kappa(M)$ of a metric space $(M, \rho)$ is the supremum of all positive real numbers $b$ such that there exists $a>1$ such that for each $x, y \in M$ and $r>0$ with $\rho(x, y)>r$ there exists $z \in M$ satisfying $\bar{B}(x, b r) \cap \bar{B}(y, a r) \subset \bar{B}(z, r) \quad[23]$. It is obvious that $\kappa(M) \geq 1$. In a Banach space $(X,\|\cdot\|)$ we denote by $\kappa_{0}(X)$ the infimum of the numbers $\kappa(C)$ where $C$ is a closed, convex, bounded and nonempty subset of $X$. It is known [2], [16] that

$$
\frac{1}{1-\delta(1)} \leq \kappa_{0}(X) \leq N(X)
$$

and $\epsilon_{0}(X)<1$ if and only $\kappa_{0}(X)>1[12]$. Therefore $\kappa_{0}(X) \leq \sqrt{2}[2]$.

Unfortunately, we know the exact value of $\kappa_{0}(X)$ or some lower bounds for $\kappa_{0}(X)$ in special spaces only [2]. Therefore it is convenient to introduce a new coefficient which plays a role similar to the one of $\kappa_{0}(X)$. In [10] T. Domínguez Benavides and H.K. Xu introduced such a new constant $\kappa_{\omega}(X)$ in Banach spaces. We give here a slightly different definition of $\kappa_{\omega}(X)$ from the one given in [10]. Namely, if $(X,\|\cdot\|)$ is a Banach space and $C$ is a nonempty bounded closed convex subset of $X$, then

(a) a number $b \geq 0$ has property $\left(P_{\omega}\right)$ with respect to $C$ if there exists some $a>1$ such that for all $x, y \in C$ and $r>0$ with $\|x-y\| \geq r$ and each weakly convergent sequence $\left\{z_{n}\right\}$ with elements in $C$ such that $\lim \sup _{n}\left\|x-z_{n}\right\| \leq$ ar and $\limsup _{n}\left\|y-z_{n}\right\| \leq b r$, there exists $z \in C$ such that $\liminf \inf _{n}\left\|z-z_{n}\right\| \leq r$

(b) $\kappa_{\omega}(C)=\sup \left\{b>0: b\right.$ has property $\left(P_{\omega}\right)$ with respect to $\left.C\right\}$;

(c) $\kappa_{\omega}(X)=\inf \left\{\kappa_{\omega}(C): C\right.$ is a nonempty bounded closed convex subset of $X\}$.

It is clear that $\kappa_{\omega}(C) \geq \kappa(C)$ for all nonempty bounded closed convex subsets $C \subset X$. Next let us observe that

$$
\kappa_{\omega}(X)=\inf \left\{\kappa_{\omega}(C): C \text { is a convex weakly compact subset of } X\right\} \text {. }
$$

Hence we get that $\kappa_{\omega}(X) \leq W C S(X)[2]$. 
Let $(M, \rho)$ be a metric space, where $M$ is not a singleton and $T: M \rightarrow M$. Then we will use the symbol $|T|$ to denote the exact Lipschitz constant of $T$, i.e.,

$$
|T|=\sup \left\{\frac{\rho(T x, T y)}{\rho(x, y)}: x, y \in M, x \neq y\right\} .
$$

Let $X$ be a Banach space, $C$ a nonempty bounded closed convex subset of $X, G$ an unbounded subset of $[0, \infty)$ such that

$$
\begin{gathered}
t+h \in G \text { for all } t, h \in G, \\
t-h \in G \text { for all } t, h \in G \text { with } t \geq h,
\end{gathered}
$$

and $\mathbf{T}=\left\{\mathfrak{T}_{\mathfrak{t}}: \mathfrak{t} \in \mathfrak{G}\right\}$ a family of self-mappings on $C$. $\mathbf{T}$ is called a semigroup of mappings on $C$ if

(i) $T_{s+t} x=T_{s} T_{t} x$ for all $s, t \in G$ and $x \in C$,

(ii) for each $x \in C$, the mapping $t \rightarrow T_{t} x$ from $G$ into $C$ is continuous when $G$ has the relative topology of $[0, \infty)$.

Let us observe that in the particular case $G=\mathbb{N}$ we get the semigroups of iterates $\mathbf{T}=\left\{\mathfrak{T}_{\mathfrak{t}}: \mathfrak{t} \in \mathfrak{G}\right\}=\left\{\mathfrak{T}_{\mathfrak{1}}^{\mathfrak{n}}: \mathfrak{n} \in \mathbb{N}\right\}$.

If $\mathbf{T}$ satisfies i. - ii. and in addition there exists $k>0$ such that

$$
\left\|T_{t} x-T_{t} y\right\| \leq k\|x-y\|
$$

for all $x, y \in C$ and $t$ in $G$, then we say that $\mathbf{T}$ is a uniformly lipschitzian ( $k$-lipschitzian) semigroup of mappings on $C$.

If $\mathbf{T}$ satisfies i. - ii. and for each $x \in C, h \in G$,

$$
\lim _{t \rightarrow \infty}\left\|T_{t+h} x-T_{t} x\right\|=0,
$$

then $\mathbf{T}$ is said to be asymptotically regular. The concept of asymptotic regularity is due to F.E. Browder and W.V. Petryshyn [5].

Let us observe that the notions of the asymptotic radius and the asymptotic center can be formulated in an obvious way for $\left\{x_{t}\right\}_{t \in G}$, where $G$ satisfies (2) and (3).

The first positive result about fixed points of uniformly lipschitzian mappings is due to K. Goebel and W.A. Kirk.

Theorem 2.1. [14] Let $X$ be a Banach space with $\epsilon_{0}(X)<1$ and let $C$ be a nonempty bounded closed convex subset of $X$. Suppose $T: C \rightarrow C$ is uniformly lipschitzian with a constant $k<\gamma$, where $\gamma>1$ satisfies the equation

$$
\gamma\left(1-\delta\left(\frac{1}{\gamma}\right)\right)=1
$$

Then $T$ has a fixed point in $C$.

By (1) it is obvious that the constant $\gamma$ from (4) is strictly less than $\kappa_{0}(X)$ [23].

In [30] K.-K. Tan and H.-K. Xu proved the following theorem which is formulated in the same spirit as the previous one. 
Theorem 2.2. [30] Let $X$ be a uniformly convex Banach space, $C$ a nonempty bounded closed convex subset of $X$ and $T: C \rightarrow C$ a k-lipschitzian mapping with $k<\gamma_{1}$, where $\gamma_{1}>1$ is the solution of the equation

$$
\gamma_{1}\left[1-\delta\left(\frac{N(X)}{\gamma_{1}^{2}}\right)\right]=1 .
$$

Then $T$ has a fixed point in $C$.

The constant $\gamma_{1}$ given by formula (5) is always bigger than the constant $\gamma$ defined by (4).

In [23] Lifshitz extended the result of Goebel and Kirk in the following way:

Theorem 2.3. [23] Let $(M, \rho)$ be a complete metric space and $T: M \rightarrow M$ a uniformly lipschitzian mapping with constant $k<\kappa(M)$. If there exists $x_{0} \in M$ such that the orbit $\left\{T^{n} x_{0}\right\}$ is bounded, then $T$ has a fixed point in $M$.

Here we must note that in the case of a Banach space we do not know in general how to compare the constant $\gamma_{1}$ given by (5) to the constant $\kappa(X)$ or $\kappa_{0}(X)$.

In each Banach space we have $\kappa_{0}(X) \leq N(X)$ (see (1)) but in particular cases we can have $\kappa_{0}(X)<\sqrt{N(X)}$ [7]. Therefore the following result is important.

Theorem 2.4. [7] Let $X$ be a Banach space $X$ with uniform normal structure and $C$ a nonempty bounded closed convex subset of $X$. If $T: C \rightarrow C$ is a uniformly $k$-lipschitzian mapping with $k<\sqrt{N(X)}$, then $T$ has a fixed point.

Now we recall a fixed point theorem in which the coefficients $\kappa_{0}(X)$ and $N(X)$ appear simultaneously.

Theorem 2.5. [9] Let $X$ be a Banach space, $C$ a nonempty bounded closed convex subset of $X$ and $T: C \rightarrow C$. If

$$
\liminf _{n}\left|T^{n}\right|<\frac{1+\sqrt{1+4 \cdot N(X) \cdot\left(\kappa_{0}(X)-1\right)}}{2},
$$

then $T$ has a fixed point.

For a discussion of this theorem see [9].

For asymptotically regular mappings we have the following results.

Theorem 2.6. [20] Let $X$ be an infinite dimensional uniformly convex Banach space, $C$ a nonempty bounded closed convex separable subset of $X$ and $T: C \rightarrow C$ an asymptotically regular mapping with $\liminf _{n}\left|T^{n}\right|<\gamma_{2}$, where $\gamma_{2}>1$ is the solution of the equation

$$
\gamma_{2}\left[1-\delta\left(\frac{W C S(X)}{\gamma_{2}^{2}}\right)\right]=1
$$

Then $T$ has a fixed point in $C$. 
Theorem 2.7. $[2,10]$ Suppose $X$ is a Banach space. Suppose also $C$ is a convex weakly compact subset of $X$ and $T: C \rightarrow C$ is asymptotically regular. If $\liminf _{n}\left|T^{n}\right|<\kappa_{\omega}(C)$, then $T$ has a fixed point.

Theorem 2.8. $[2,10]$ Suppose $X$ is a Banach space such that $W C S(X)>$ $1, C$ is a convex weakly compact subset of $X$ and $T: C \rightarrow C$ is asymptotically regular. If $\liminf _{n}\left|T^{n}\right|<\sqrt{W C S(X)}$, then $T$ has a fixed point.

Theorem 2.9. [9] Let $X$ be a reflexive Banach space, $C$ a nonempty bounded closed convex subset of $X$ and $T: C \rightarrow C$ an asymptotically regular mapping. If

$$
\liminf _{n}\left|T^{n}\right|<\frac{1+\sqrt{1+4 \cdot W C S(X) \cdot\left(\kappa_{\omega}(X)-1\right)}}{2},
$$

then $T$ has a fixed point.

For a discussion of the connections among the above results see $[9,10]$.

In [11], [20], [21], [30], [31] and [32] some of the above results were reformulated in terms of semigroups. It is also worthwhile to see [4], [15], [18], [19], [22], [24], [25], [26], [27], [28], [29], [33], [34].

\section{EXISTENCE OF FIXED POINTS OF LIPSCHITZIAN SEMIGROUPS OF MAPPINGS}

We begin with a generalization of Theorem 2.2.

Theorem 3.1. Let $(X,\|\cdot\|)$ be a Banach space with $\epsilon_{0}<1, C$ a nonempty bounded closed convex subset of $X$ and $\mathbf{T}=\left\{\mathfrak{T}_{\mathfrak{t}}: \mathfrak{t} \in \mathfrak{G}\right\}$ a uniformly lipschitzian semigroup of mappings on $C$ with $\sup _{t}\left|T_{t}\right|=k<\bar{\gamma}$, where $\bar{\gamma}>1$ is the solution of the equation

$$
\bar{\gamma}\left[1-\delta\left(\frac{N(X)}{\bar{\gamma}}\right)\right]=1
$$

Then there exists a common fixed point of $\mathbf{T}$.

Proof. Without loss of generality we can assume that $(X,\|\cdot\|)$ is uniformly convex and $k>1$. If $0<\epsilon_{0}<1$ we need only make minor changes in the following proof. Let us denote $N(X)$ by $N$. For each $x \in C$ let $z$ be the unique element of $A c\left(C,\left\{T_{t} x\right\}\right)$. Let us assume that

$$
\frac{d(x)}{N}=\frac{\operatorname{diam}_{a}\left\{T_{t} x\right\}}{N} \geq r=r\left(C,\left\{T_{t} x\right\}\right)>0
$$

and $d(z)=\operatorname{diam}_{a}\left\{T_{t} z\right\}>0$. Then we can find sequences $\left\{s_{n}\right\}$ and $\left\{t_{n}\right\}$ such that

and

$$
\lim _{n} s_{n}=\lim _{n} t_{n}=\infty
$$

$$
\lim _{n}\left\|T_{t_{n}} z-T_{s_{n}} z\right\|=\operatorname{diam}_{a}\left\{T_{t} z\right\}=d(z) .
$$

Next we have

$$
r_{a}\left(T_{s_{n}} z,\left\{T_{t} x\right\}\right) \leq k \cdot r
$$


and

$$
r_{a}\left(T_{t_{n}} z,\left\{T_{t} x\right\}\right) \leq k \cdot r
$$

Hence

$$
\begin{gathered}
r \leq \liminf _{n} r_{a}\left(\frac{T_{s_{n}} z+T_{t_{n}} z}{2},\left\{T_{t} x\right\}\right) \leq\left[1-\delta\left(\frac{d(z)}{k \cdot r}\right)\right] \cdot k \cdot r, \\
1 \leq\left[1-\delta\left(\frac{d(z)}{k \cdot r}\right)\right] \cdot k \leq\left[1-\delta\left(\frac{N \cdot d(z)}{d(x) \cdot k}\right)\right] \cdot k,
\end{gathered}
$$

and finally

$$
d(z) \leq\left[\frac{k}{N} \cdot \delta^{-1}\left(1-\frac{1}{k}\right)\right] \cdot d(x) .
$$

Let us denote $\frac{k}{N} \cdot \delta^{-1}\left(1-\frac{1}{k}\right)$ by $a$. Directly from our assumption we get $a<1$. Now we define a sequence $\left\{x_{n}\right\}_{n=0}^{\infty}$ in $C$ in the following way: $x_{0}$ is an arbitrarily chosen element of $C$ and $x_{n+1}$ is the unique element of $A c\left(C,\left\{T_{t} x_{n}\right\}\right)$ for $n=0,1,2, \ldots$. By (9) we obtain

$$
d\left(x_{n}\right) \leq a^{n} \cdot d\left(x_{0}\right),
$$

and the inequalities

$$
\begin{gathered}
\left\|x_{n}-x_{n+1}\right\| \leq\left\|x_{n}-T_{j} x_{n-1}\right\|+\left\|T_{j} x_{n-1}-T_{i} x_{n}\right\|+\left\|T_{i} x_{n}-x_{n+1}\right\| \\
\leq\left\|x_{n}-T_{j} x_{n-1}\right\|+k\left\|T_{j-i} x_{n-1}-x_{n}\right\|+\left\|T_{i} x_{n}-x_{n+1}\right\|
\end{gathered}
$$

which are valid for $i<j$, lead to

$$
\left\|x_{n}-x_{n+1}\right\| \leq(1+k) d\left(x_{n-1}\right)+d\left(x_{n}\right) \leq\left[(1+k) a^{n-1}+a^{n}\right] \cdot d\left(x_{0}\right) .
$$

This, in turn, yields the conclusion that $\left\{x_{n}\right\}_{n=0}^{\infty}$ is norm Cauchy and hence strongly convergent. Let $x=\lim _{n} x_{n}$. Then by (8) for each $s \in G$ we have

$$
\begin{aligned}
\left\|x-T_{s} x\right\| & \leq \lim _{n}\left\|x-x_{n+1}\right\|+\limsup _{n} \limsup _{t}\left\|x_{n+1}-T_{t+s} x_{n}\right\| \\
+\limsup & \limsup _{t}\left\|T_{t+s} x_{n}-T_{s} x_{n+1}\right\|+\lim _{n}\left\|T_{s} x_{n+1}-T_{s} x\right\| \\
& \leq \lim _{n}(1+k)\left[\left\|x-x_{n+1}\right\|+\frac{d\left(x_{n}\right)}{N}\right]=0 .
\end{aligned}
$$

This completes the proof.

Remark 3.1. The constant $\bar{\gamma}$ given by (7) is bigger than the constant $\gamma$ defined by (5).

For our next results we need the following simple fact.

Lemma 3.1. Let $(X,\|\cdot\|)$ be a Banach space.

1) For each $0<\frac{1}{\theta}<w$-SOC $(X)$ and every asymptotically regular sequence $\left\{x_{n}\right\}$ with a weakly compact $\overline{c o n v}\left\{x_{n}\right\}$ there exists a weakly convergent to $w$ subsequence $\left\{x_{n_{i}}\right\}$ such that

(i) $r\left(w,\left\{x_{n_{i}}\right\}\right) \leq \theta \cdot \operatorname{diam}_{a}\left(\left\{x_{n}\right\}\right)$,

(ii) $\|w-y\| \leq r_{a}\left(y,\left\{x_{n_{i}}\right\}\right)$ for every $y \in X$. 
2) For each $0<\frac{1}{\theta}<w-A N(X)$ and every asymptotically regular sequence $\left\{x_{n}\right\}$ with a weakly compact $\overline{c o n v}\left\{x_{n}\right\}$ there exists a subsequence $\left\{x_{n_{i}}\right\}$ and a point $w \in \overline{\operatorname{conv}}\left\{x_{n}\right\}$ such that

$$
r\left(w,\left\{x_{n_{i}}\right\}\right) \leq \theta \cdot \operatorname{diam}_{a}\left(\left\{x_{n}\right\}\right) .
$$

ProOF. 1) ii. It is sufficient to apply the lower semicontinuity of $\|\cdot\|$ with respect to the weak topology because we have

$$
r_{a}\left(y,\left\{x_{n_{i}}\right\}\right)=\limsup _{i}\left\|y-x_{n_{i}}\right\|
$$

and

$$
y-x_{n_{i}} \rightarrow y-w .
$$

We will use the above lemma in the proofs of the next three theorems.

Theorem 3.2. Let $(X,\|\cdot\|)$ be an infinite dimensional Banach space with $\epsilon_{0}<1, C$ a nonempty bounded closed convex subset of $X$ and $\mathbf{T}=\left\{\mathfrak{T}_{\mathfrak{t}}: \mathfrak{t} \in \mathfrak{G}\right\}$ a uniformly $k$-lipschitzian asymptotically regular semigroup of mappings on $C$ with $k<\overline{\bar{\gamma}}$, where $\overline{\bar{\gamma}}$ satisfies

$$
\overline{\bar{\gamma}}^{2}>\max \left(1, \frac{w-A N(X)}{2}\right)
$$

and is the solution of the equation

$$
\overline{\bar{\gamma}}\left[1-\delta\left(\frac{w-A N(X)}{(\overline{\bar{\gamma}})^{2}}\right)\right]=1 .
$$

Then there exists in $C$ a common fixed point of $\mathbf{T}$.

ProOf. Without loss of generality we can assume that $(X,\|\cdot\|)$ is uniformly convex, $k^{2}>\frac{w-A N(X)}{2}$ and $k>1$. If $0<\epsilon_{0}<1$ we need only make minor changes in our proof. First we choose $\theta$ such that $1<\frac{1}{\theta}<w-A N(X)$ and

$$
k\left[1-\delta\left(\frac{1}{\theta k^{2}}\right)\right]<1 .
$$

Next we fix $t_{0}, h \in G$ with $h>0$ and define $t_{n}=t_{0}+n h$ for $n=1,2, \ldots$ Let us observe that for each $x \in C$ the sequence $\left\{T_{t_{n}} x\right\}$ is asymptotically regular and by Lemma 3.1 for each $x \in C$ there exists a weakly convergent subsequence $\left\{T_{t_{n_{i}}} x\right\}$ with $w \in \overline{\operatorname{conv}}\left\{T_{t_{n}} x\right\}$ such that

$$
r_{a}\left(w,\left\{T_{t_{n_{i}}} x\right\}\right) \leq \theta \cdot \operatorname{diam}_{a}\left(\left\{T_{t_{n}} x\right\}\right) .
$$

In other words, for each $x \in C$ we can find a subsequence $\left\{T_{t_{n_{i}}} x\right\}$ and $w(x) \in C$ which satisfy the inequality (11). Hence, if $z(x)$ is the unique element of $A c\left(C,\left\{T_{t_{n_{i}}} x\right\}\right)$, then we have

$$
r(x)=r_{a}\left(z(x),\left\{T_{t_{n_{i}}} x\right\}\right) \leq r_{a}\left(w(x),\left\{T_{t_{n_{i}}} x\right\}\right) \leq \theta \cdot \operatorname{diam}_{a}\left(\left\{T_{t_{n}} x\right\}\right) .
$$


The asymptotic regularity of $\mathbf{T}$ leads to

$$
\begin{aligned}
r_{a}\left(T_{t_{m}} z(x),\left\{T_{t_{n_{i}}} x\right\}\right) \leq & r_{a}\left(T_{t_{m}} z(x),\left\{T_{t_{n_{i}}+t_{m}} x\right\}\right) \\
& +\underset{i}{\limsup }\left\|T_{t_{n_{i}}+t_{m}} x-T_{t_{n_{i}}} x\right\| \\
= & r_{a}\left(T_{t_{m}} z(x),\left\{T_{t_{n_{i}}+t_{m}} x\right\}\right) \leq k \cdot r_{a}\left(z(x),\left\{T_{t_{n_{i}}} x\right\}\right)
\end{aligned}
$$

for every $m$. Now we set

$$
R(x)=r_{a}\left(x,\left\{T_{t_{n}} x\right\}\right)=\limsup _{n}\left\|x-T_{t_{n}} x\right\| .
$$

We observe that by the asymptotic regularity of $\mathbf{T}$ and since

$$
t_{l}=t_{0}+l h \quad \text { for } l=1,2, \ldots,
$$

we have

$$
\begin{gathered}
\operatorname{diam}_{a}\left(\left\{T_{t_{n}} x\right\}\right)=\lim _{\tilde{m}} \sup _{\tilde{m}<m<n}\left\|T_{t_{m}} x-T_{t_{n}} x\right\| \\
=\lim _{\tilde{m}} \sup _{\tilde{m}<m}\left(\max _{1 \leq n \leq n_{0}}\left\|T_{t_{m}} x-T_{t_{m+n}} x\right\|, \sup _{l>0}\left\|T_{t_{m}} x-T_{t_{m+n}+l} x\right\|\right) \\
=\lim _{\tilde{m}}\left[\sup _{\tilde{m}<m}\left(\sup _{l>0}\left\|T_{t_{m}} x-T_{t_{m+n_{0}+l}} x\right\|\right)\right] \\
\leq \limsup _{\tilde{m}}\left(\sup _{\tilde{m}<m}\left\|T_{t_{m}} x-T_{m h} x\right\|\right) \\
\quad+\limsup _{\tilde{m}}\left[\sup _{\tilde{m}<m}\left(\sup _{l>0}\left\|T_{m h} x-T_{t_{m+n_{0}+l}} x\right\|\right)\right] \\
\leq k \cdot \sup _{l>0}\left\|x-T_{t_{n_{0}+l}} x\right\|=k \cdot \sup _{n>n_{0}}\left\|x-T_{t_{n}} x\right\|
\end{gathered}
$$

for $n_{0} \geq 1$. Hence

$$
\operatorname{diam}_{a}\left(\left\{T_{t_{n}} x\right\}\right) \leq k \cdot \limsup _{n}\left\|x-T_{t_{n}} x\right\|=k \cdot R(x) .
$$

We construct the sequence $\left\{x_{m}\right\}$ in the following way: $x_{0} \in C$ is arbitrary and

$$
x_{m+1}=z\left(x_{m}\right)
$$

for $m=0,1, \ldots$. By (12) and (14) we get

$$
r_{m}=r\left(x_{m}\right) \leq \theta \cdot k \cdot R\left(x_{m}\right) .
$$

Now we have to consider two cases. If for some $m_{0}$ we have $r_{m_{0}}=0$, then we get

$$
\begin{gathered}
R\left(z\left(x_{m_{0}}\right)\right)=\limsup _{n}\left\|z\left(x_{m_{0}}\right)-T_{t_{n}} z\left(x_{m_{0}}\right)\right\| \\
\leq \limsup _{n} \limsup _{i}\left[\left\|z\left(x_{m_{0}}\right)-T_{t_{n_{i}}} x_{m_{0}}\right\|\right. \\
\left.+\left\|T_{t_{n_{i}}} x_{m_{0}}-T_{t_{n}+t_{n_{i}}} x_{m_{0}}\right\|+\left\|T_{t_{n}+t_{n_{i}}} x_{m_{0}}-T_{t_{n}} z\left(x_{m_{0}}\right)\right\|\right]=0 .
\end{gathered}
$$


In the second case we have $r_{m}>0$ for all $m \geq 0$. For each $m$ we first choose an arbitrary $0<\epsilon<R\left(x_{m+1}\right)$ and then $j$ such that

$$
\left\|T_{t_{j}} x_{m+1}-x_{m+1}\right\| \geq R\left(x_{m+1}\right)-\epsilon .
$$

Now we find $i_{0}$ such that for all $i \geq i_{0}$ we have

$$
\left\|T_{t_{n_{i}}} x_{m}-x_{m+1}\right\| \leq r_{m}+\frac{\epsilon}{2}
$$

(the subsequence $\left\{t_{n_{i}}\right\}$ depends on $x_{m}$ here) and (see (13))

$$
\left\|T_{t_{n_{i}}} x_{m}-T_{t_{j}} x_{m+1}\right\| \leq k \cdot\left(r_{m}+\epsilon\right) .
$$

It follows that

$$
\begin{gathered}
\left\|T_{t_{n_{i}} x_{m}-\frac{1}{2}\left(x_{m+1}+T_{t_{j}} x_{m+1}\right) \|}\right\| \\
\leq k \cdot\left(r_{m}+\epsilon\right) \cdot\left[1-\delta\left(\frac{R\left(x_{m+1}\right)-\epsilon \cdot}{k \cdot\left(r_{m}+\epsilon\right)}\right)\right] .
\end{gathered}
$$

Letting $i$ tend to infinity we obtain

$$
r_{m} \leq k \cdot\left(r_{m}+\epsilon\right) \cdot\left[1-\delta\left(\frac{R\left(x_{m+1}\right)-\epsilon}{k \cdot\left(r_{m}+\epsilon\right)}\right)\right] .
$$

Taking now $\epsilon$ to 0 we get

$$
r_{m} \leq k \cdot r_{m} \cdot\left[1-\delta\left(\frac{R\left(x_{m+1}\right)}{k \cdot r_{m}}\right)\right]
$$

which after applying the inequality (15) implies that

$$
R\left(x_{m+1}\right) \leq\left[\theta \cdot k^{2} \cdot \delta^{-1}\left(1-\frac{1}{k}\right)\right] \cdot R\left(x_{m}\right) .
$$

Let us observe that

$$
0<\alpha=\theta \cdot k^{2} \cdot \delta^{-1}\left(1-\frac{1}{k}\right)<1
$$

and therefore

$$
R\left(x_{m}\right) \leq \alpha^{m} \cdot R\left(x_{0}\right)
$$

for $m=1,2, \ldots$. Hence by (15) and (16) we deduce that

$$
\begin{gathered}
\left\|x_{m+1}-x_{m}\right\| \leq \limsup _{i}\left\|x_{m+1}-T_{t_{n_{i}}} x_{m}\right\|+\underset{i}{\limsup }\left\|T_{t_{n_{i}}} x_{m}-x_{m}\right\| \\
\leq r_{a}\left(x_{m+1},\left\{T_{t_{n_{i}}} x_{m}\right\}\right)+R\left(x_{m}\right)=r_{m}+R\left(x_{m}\right) \\
\leq(\theta \cdot k+1) \cdot R\left(x_{m}\right) \leq(\theta \cdot k+1) \cdot \alpha^{m} \cdot R\left(x_{0}\right)
\end{gathered}
$$

(the subsequence $\left\{t_{n_{i}}\right\}$ depends on $x_{m}$ here) and therefore the sequence $\left\{x_{n}\right\}$ is strongly convergent to some $x \in C$. By the inequality

$$
|R(x)-R(y)| \leq(1+k) \cdot\|x-y\|,
$$

which is valid for all $x, y \in C$, we have $R(x)=0$.

Thus in both cases we can find $y \in C$ with $R(y)=0$. This means that

$$
y=\lim _{n} T_{t_{n}} y
$$


and by the asymptotic regularity of $\mathbf{T}$ we have

$$
T_{t} y=\lim _{n} T_{t+t_{n}} y=\lim _{n} T_{t_{n}} y=y
$$

for each $t \in G$. This completes the proof.

Theorem 3.3. Let $C$ be a convex weakly compact subset of a Banach space $X$ with $w$-SOC $(X)>1$. Then every asymptotically regular uniformly $k$-lipschitzian semigroup $\mathbf{T}=\left\{\mathfrak{T}_{\mathfrak{t}}: \mathfrak{t} \in \mathfrak{G}\right\}$ of mappings on $C$ with $k<$ $[w-S O C(X)]^{\frac{1}{2}}$ has a common fixed point.

Proof. Let $k \geq 1$ and let us fix $\theta$ such that

$$
k^{2}<\frac{1}{\theta}<w-S O C(X) .
$$

We choose $t_{0}, h \in G$ with $h>0$ and consider the sequence $\left\{t_{n}\right\}=\left\{t_{0}+n h\right\}$. For each $x \in C$ we define $R(x)$ by

$$
R(x)=r_{a}\left(x,\left\{T_{t_{n}} x\right\}\right)=\limsup _{n}\left\|x-T_{t_{n}} x\right\| .
$$

Let us observe that for each $x \in C$ the sequence $\left\{T_{t_{n}} x\right\}$ is asymptotically regular and by Lemma 3.1 for each $x \in C$ there is a weakly convergent to $w$ subsequence $\left\{T_{t_{n_{i}}} x\right\}$ such that

(i) $r_{a}\left(w,\left\{T_{t_{n_{i}}} x\right\}\right) \leq \theta \cdot \operatorname{diam}_{a}\left(\left\{T_{t_{n}} x\right\}\right)$,

(ii) $\|y-w\| \leq r_{a}\left(y,\left\{T_{t_{n_{i}}} x\right\}\right)$ for every $y \in X$.

By i., ii., (14) and the asymptotic regularity of $\mathbf{T}$ we obtain

$$
\begin{gathered}
r_{a}\left(T_{t_{m}} w,\left\{T_{t_{n_{i}}} x\right\}\right) \leq r_{a}\left(T_{t_{m}} w,\left\{T_{t_{n_{i}}+t_{m}} x\right\}\right)+\underset{i}{\limsup }\left\|T_{t_{n_{i}}+t_{m}} x-T_{t_{n_{i}}} x\right\| \\
\leq k \cdot r_{a}\left(w,\left\{T_{t_{n_{i}}} x\right\}\right) \leq k \cdot \theta \cdot \operatorname{diam}_{a}\left(\left\{T_{t_{n}} x\right\}\right) \leq \theta \cdot k^{2} \cdot R(x), \\
\left\|T_{t_{m}} w-w\right\| \leq r_{a}\left(T_{t_{m}} w,\left\{T_{t_{n_{i}}} x\right\}\right) \leq \theta \cdot k^{2} \cdot R(x)
\end{gathered}
$$

for each $m$, and finally

$$
R(w) \leq \theta \cdot k^{2} \cdot R(x)=\alpha \cdot R(x),
$$

where $0 \leq \alpha=\theta \cdot k^{2}<1$. Consequently, for each $x \in C$ there is $w(x)$ such that

and

$$
\begin{gathered}
T_{t_{n_{i}}} x \rightarrow w(x), \\
r_{a}\left(w(x),\left\{T_{t_{n_{i}}} x\right\}\right) \leq \theta \cdot k \cdot R(x)
\end{gathered}
$$

$$
R(w) \leq \alpha \cdot R(x)
$$

This allows us to construct a sequence $\left\{x_{m}\right\}$ which is convergent to a fixed point of $\mathbf{T}$. We simply choose the first element $x_{1}$ arbitrarily and next we set

$$
x_{m}=w\left(x_{m-1}\right)
$$

for $m=2,3, \ldots$. Now it is sufficient to observe that

$$
R\left(x_{m}\right) \leq \alpha^{m-1} \cdot R\left(x_{1}\right)
$$


and repeat the arguments from the end of the proof of Theorem 3.2 to finish the present proof.

In the next theorem we employ $\kappa_{\omega}(X)$ and $w$-SOC $(X)$.

Theorem 3.4. Let $(X,\|\cdot\|)$ be a Banach space with $w$-SOC $(X)>1, C$ a convex weakly compact subset of $X$ and $\mathfrak{T}=\left\{\mathfrak{T}_{\mathfrak{t}}: \mathfrak{t} \in \mathfrak{G}\right\}$ an asymptotically regular uniformly $k$-lipschitzian semigroup of mappings on $C$. If

$$
k<\frac{1+\sqrt{1+4 \cdot[w-S O C(X)] \cdot\left(\kappa_{\omega}(X)-1\right)}}{2},
$$

then $\mathbf{T}$ has a common fixed point.

Proof. The proof is based on ideas presented in [9]. Let us denote $S=w$ $S O C(X)$ and $\kappa=\kappa_{\omega}(X)$. Without loss of generality we may assume that $S<+\infty$ (see Theorem 3.2 in [6]) and $k>1$. The inequality (17) and $k>1$ imply $\kappa>1$. Next we observe that

$$
\frac{1+\sqrt{1+4 \cdot S \cdot(\kappa-1)}}{2} \leq S<+\infty,
$$

because (see Section 2)

$$
\kappa \leq W C S(X) \leq S<+\infty .
$$

The inequality

$$
k<\frac{1+\sqrt{1+4 \cdot S \cdot(\kappa-1)}}{2}
$$

implies the inequality

$$
\frac{k}{S}<\frac{\kappa-1}{k-1} .
$$

Directly from the definition of $\kappa$ we find $a>1$ and $1<b<\kappa$ such that

$$
\frac{k}{S}<\frac{\frac{b}{a}-1}{k-1},
$$

and for all $x, y \in C$ and $r>0$ with $\|x-y\| \geq r$ and each weakly convergent sequence $\left\{z_{n}\right\}$ in $C$ for which $\lim \sup _{n}\left\|x-z_{n}\right\| \leq a \cdot r$ and $\lim \sup _{n}\left\|y-z_{n}\right\| \leq$ $b \cdot r$ there exists some $z \in C$ such that $\liminf _{n}\left\|z-z_{n}\right\| \leq r$. Next we choose $\epsilon>0$ such that

$$
\frac{1+2 \epsilon}{a}=\alpha<1
$$

Similarly as in the proofs of the previous theorems we consider the sequence $\left\{t_{n}\right\}=\left\{t_{0}+n h\right\}$, where $t_{0}, h \in G$ and $h>0$. For $x \in C$ we define $\bar{R}(x)$ as follows

$$
\bar{R}(x)=\inf \left\{r>0: \exists_{y \in C} \liminf _{n}\left\|x-T_{t_{n}} y\right\| \leq r\right\} .
$$

First we will show that $\bar{R}(\widetilde{x})=0$ for some $\widetilde{x} \in C$. To this end we take an arbitrary $x \in C$. Assume that $\bar{R}(x)>0$. Then we can find $y \in C$ with

$$
\liminf _{n}\left\|x-T_{t_{n}} y\right\|<\bar{R}(x) \cdot(1+\epsilon) .
$$


There are two possibilities; either

$$
\sup _{n}\left\|x-T_{t_{n}}(x)\right\| \leq \frac{S \cdot \bar{R}(x) \cdot(1+\epsilon)}{k \cdot a}
$$

or

$$
\left\|x-T_{t_{j}}(x)\right\|>\frac{S \cdot \bar{R}(x) \cdot(1+\epsilon)}{k \cdot a}
$$

for some $j$.

Let us take a look at the first case. By (14) and (19) we get

$$
\operatorname{diam}_{a}\left(\left\{T_{t_{n}} x\right\}\right) \leq k \cdot \limsup _{n}\left\|x-T_{t_{n}}(x)\right\| \leq \frac{S \cdot \bar{R}(x) \cdot(1+\epsilon)}{a}
$$

and after applying the definition of $w$-SOC $(X)$ we obtain

$$
\begin{gathered}
S \cdot \inf \left\{r_{a}\left(w,\left\{T_{t_{n_{i}}} x\right\}\right):\left\{T_{t_{n_{i}}} x\right\} \text { is weakly convergent to } w\right\} \\
\leq \frac{S \cdot \bar{R}(x) \cdot(1+\epsilon)}{a} .
\end{gathered}
$$

This implies that

$\inf \left\{r_{a}\left(w,\left\{T_{t_{n_{i}}} x\right\}\right):\left\{T_{t_{n_{i}}} x\right\}\right.$ is weakly convergent to $\left.w\right\} \leq \frac{\bar{R}(x) \cdot(1+\epsilon)}{a}$. Hence there exists a subsequence $\left\{T_{t_{n_{i}}} x\right\}$ which weakly converges to $w \in C$ such that

$$
\lim _{i}\left\|w-T_{t_{n_{i}}} x\right\|<\frac{\bar{R}(x) \cdot(1+2 \epsilon)}{a}=\alpha \bar{R}(x)
$$

and therefore

$$
\bar{R}(w)<\alpha \bar{R}(x)
$$

Next we see that

$$
\|w-x\| \leq\left\|w-T_{t_{n_{i}}} x\right\|+\left\|x-T_{t_{n_{i}}} x\right\|
$$

and by (19) and (21) this yields

$$
\begin{aligned}
&\|w-x\| \leq \lim _{i}\left\|w-T_{t_{n_{i}}} x\right\|+\limsup _{i}\left\|x-T_{t_{n_{i}}} x\right\| \\
& \leq \alpha \cdot \bar{R}(x)+\frac{S \cdot \bar{R}(x) \cdot(1+\epsilon)}{k \cdot a} \\
& \leq \alpha \cdot \bar{R}(x)+\frac{S \cdot \alpha \cdot \bar{R}(x)}{k}=\alpha \cdot\left(1+\frac{S}{k}\right) \cdot \bar{R}(x) .
\end{aligned}
$$

Let us now consider the second case. By (20) we have

$$
\left\|x-T_{t_{j}} x\right\|>\frac{S \cdot \bar{R}(x) \cdot(1+\epsilon)}{k \cdot a}
$$

for some $j$. Let us choose a weakly convergent $\left\{T_{t_{n_{i}}} y\right\}$ such that

$$
\left\|x-T_{t_{n_{i}}} y\right\|<\bar{R}(x) \cdot\left(1+\frac{\epsilon}{2}\right)
$$


and

for each $i$. This implies

$$
\sum_{m=1}^{t_{j}}\left\|T_{t_{n_{i}+m-1}} y-T_{t_{n_{i}+m}} y\right\|<\bar{R}(x) \cdot \frac{\epsilon}{2}
$$

$$
\left\|x-T_{t_{n_{i}}+t_{j}} y\right\|<\left\|x-T_{t_{n_{i}}} y\right\|+\sum_{m=1}^{t_{j}}\left\|T_{t_{n_{i}+m-1}} y-T_{t_{n_{i}+m}} y\right\|<\bar{R}(x) \cdot(1+\epsilon)
$$

and

$$
\left\|T_{t_{j}} x-T_{t_{n_{i}}+t_{j}} y\right\| \leq k \cdot \bar{R}(x) \cdot(1+\epsilon)
$$

for every $i$. By (18) we can choose $\lambda$ such that

$$
\frac{k}{S}<\lambda<\frac{\frac{b}{a}-1}{k-1}
$$

Then by (24), (25) and (26) we have

$$
\begin{gathered}
\left\|\lambda T_{t_{j}} x+(1-\lambda) x-T_{t_{n_{i}}+t_{j}} y\right\| \\
\leq \lambda\left\|T_{t_{j}} x-T_{t_{n_{i}}+t_{j}} y\right\|+(1-\lambda)\left\|x-T_{t_{n_{i}}+t_{j}} y\right\| \\
\leq \lambda \cdot k \cdot \bar{R}(x) \cdot(1+\epsilon)+(1-\lambda) \cdot \bar{R}(x) \cdot(1+\epsilon) \\
=[\lambda(k-1)+1] \cdot \bar{R}(x) \cdot(1+\epsilon)<b \cdot \frac{\bar{R}(x) \cdot(1+\epsilon)}{a}
\end{gathered}
$$

and next by (20) and (26),

$$
\begin{aligned}
& \left\|\lambda T_{t_{j}} x+(1-\lambda) x-x\right\|=\lambda\left\|T_{t_{j}} x-x\right\| \\
& >\lambda \cdot \frac{S \cdot \bar{R}(x) \cdot(1+\epsilon)}{k \cdot a}>\frac{\bar{R}(x) \cdot(1+\epsilon)}{a} .
\end{aligned}
$$

Directly from the definition of $b$ and by (24), (27) and (28) there exists $w \in C$ such that

$$
\left\|w-T_{t_{n_{i}}+t_{j}} y\right\| \leq \frac{\bar{R}(x) \cdot(1+\epsilon)}{a} \leq \alpha \cdot \bar{R}(x)
$$

for every $i$. Therefore by the asymptotic regularity of $\mathbf{T}$ and (29) we get

$$
\begin{gathered}
\bar{R}(w) \leq \limsup _{i}\left\|w-T_{t_{n_{i}}} y\right\| \\
\leq \limsup _{i}\left\|w-T_{t_{n_{i}}+t_{j}} y\right\|+\limsup _{i}\left\|T_{t_{n_{i}}+t_{j}} y-T_{t_{n_{i}}} y\right\| \\
=\limsup _{i}\left\|w-T_{t_{n_{i}}+t_{j}} y\right\| \leq \alpha \cdot \bar{R}(x),
\end{gathered}
$$

and by (24) and (29) we obtain

$$
\begin{gathered}
\|w-x\| \leq\left\|w-T_{t_{n_{i}}+t_{j}} y\right\|+\left\|x-T_{t_{n_{i}}+t_{j}} y\right\| \\
\leq \alpha \cdot \bar{R}(x)+\bar{R}(x) \cdot(1+\epsilon)=(1+\epsilon+\alpha) \cdot \bar{R}(x) .
\end{gathered}
$$


Thus in both cases for every $x \in C$ we can find $w \in C$ for which the following inequalities are valid:

$$
\bar{R}(w) \leq \alpha \cdot \bar{R}(x)
$$

and

$$
\|w-x\| \leq \max \left\{1+\epsilon+\alpha, \alpha \cdot\left(1+\frac{S}{k}\right)\right\} \cdot \bar{R}(x)=M \cdot \bar{R}(x)
$$

(by (22), (23), (30) and (31)). This allows us to define a function $f: C \rightarrow C$ by $f(x)=w$, where $w$ satisfies (32) and (33). We introduce a sequence $\left\{x_{n}\right\}$ as follows: $x_{0}$ is chosen in an arbitrary way and next $x_{n}=f\left(x_{n-1}\right)$ for $n=1,2, \ldots$. The sequence $\left\{x_{n}\right\}$ is a Cauchy sequence. Indeed, the inequalities (32) and (33) lead to

$$
\left\|x_{n}-x_{n-1}\right\| \leq M \cdot \bar{R}\left(x_{n-1}\right) \leq M \cdot \alpha^{n-1} \cdot \bar{R}\left(x_{0}\right) .
$$

Setting $\widetilde{x}$ as the limit of $\left\{x_{n}\right\}$ and applying the inequality

$$
|\bar{R}(u)-\bar{R}(v)| \leq\|u-v\|,
$$

which is valid for all $u, v \in C$, we get $\bar{R}(\widetilde{x})=0$.

Now we prove that $\bar{R}(\widetilde{x})=0$ implies that $x$ is a common fixed point of $\mathbf{T}$. To prove this fact it is sufficient to observe that for every $y \in C, t \in G$ and every natural $n$ we have

$$
\begin{aligned}
\left\|\widetilde{x}-T_{t} \widetilde{x}\right\| & \leq\left\|\widetilde{x}-T_{t_{n}} y\right\|+\left\|T_{t_{n}} y-T_{t_{n}+t} y\right\|+\left\|T_{t_{n}+t} y-T_{t} \widetilde{x}\right\| \\
& \leq(1+k)\left\|\widetilde{x}-T_{t_{n}} y\right\|+\left\|T_{t_{n}} y-T_{t_{n}+t} y\right\| .
\end{aligned}
$$

Applying the definition of $\bar{R}$ and the asymptotic regularity of $\mathbf{T}$ we complete the proof.

\section{EXAmples}

Example 4.1. Let us consider the space $X_{\beta}^{p}[6]$, where $1<p<\infty$ and $1<\beta<4^{\frac{1}{p}}$. Then for $2^{\frac{1}{p}} \leq \beta<4^{\frac{1}{p}}$ we have $\kappa_{\omega}\left(X_{\beta}^{p}\right)=W C S\left(X_{\beta}^{p}\right)=$ $N\left(X_{\beta}^{p}\right)=1[2]$, but $w-A N\left(X_{\beta}^{p}\right)=w-S O C\left(X_{\beta}^{p}\right)=\frac{4^{\frac{1}{p}}}{\beta}>1[6]$.

Example 4.2. Let us observe that for the space $X_{\beta}^{2}$ with $1<\beta<\frac{\sqrt{5}}{2}$, we get $W C S\left(X_{\beta}^{2}\right)=\max \left(1, \frac{\sqrt{2}}{\beta}\right)[2,3], w-A N\left(X_{\beta}^{2}\right)=w-S O C\left(X_{\beta}^{2}\right)=\sqrt{2}[6]$ and $\kappa_{\omega}(X) \geq \kappa_{0}(X)[10]$, where

$$
\kappa_{0}\left(X_{\beta}^{2}\right)=\left(1+\frac{1}{\beta^{2}}-\frac{2}{\beta^{2}} \sqrt{\beta^{2}-1}\right)^{\frac{1}{2}}
$$

[9] (see also [35]). Hence for $\beta$ sufficiently close to 1 the constants

$$
\frac{1+\sqrt{1+4 \cdot W C S\left(X_{\beta}^{2}\right) \cdot\left(\kappa_{0}\left(X_{\beta}^{2}\right)-1\right)}}{2}
$$




$$
<\frac{1+\sqrt{1+4 \cdot\left[w-S O C\left(X_{\beta}^{2}\right)\right] \cdot\left(\kappa_{0}\left(X_{\beta}^{2}\right)-1\right)}}{2}
$$

are strictly bigger than $\sqrt{w-S O C\left(X_{\beta}^{2}\right)}=2^{\frac{1}{4}}$. Let us observe in addition that $[16]$

$$
\epsilon_{0}\left(X_{\beta}^{2}\right)= \begin{cases}2\left(\beta^{2}-1\right)^{\frac{1}{2}} & \text { for } 1<\beta \leq \sqrt{2} \\ 2 & \text { for } \sqrt{2}<\beta<2\end{cases}
$$

and therefore for $1<\beta<\frac{\sqrt{5}}{2}$ the constant $\overline{\bar{\gamma}}$ given by (10) is strictly bigger than the constant $\gamma_{2}$ defined by (6).

\section{ACKNOWLEDGMENTS}

The third author was partially supported by the Fund for the Promotion of Research at the Technion. Part of the work on this paper was done when the third author visited the Institute of Mathematics at UMCS. He thanks the Institute and its members for their hospitality.

All the authors thank the referee for several useful comments and corrections.

\section{REFERENCES}

[1] A. G. AKSOY and M. A. KHAMSI, Nonstandard methods in fixed point theory, Springer-Verlag, New York, 1990.

[2] J. M. AYERBE TOLEDANO, T. DOMÍNGUEZ BENAVIDES and G. LOPEZ ACEDO, Measures of noncompactness in metric fixed point theory, Birkhäuser, Basel, 1997.

[3] J. M. AYERBE and H.-K. XU, On certain geometric coefficients of Banach spaces related to fixed point theory, Panamer. Math. J. 3 (1993), 47-59.

[4] J.-B. BAILLON, Quelques aspects de la théorie des points fixes dans les espaces de Banach, I, Séminaire d'Analyse Fonctionnelle de l'Ecole Polytechnique, Paris, no. VII, 1978-1979.

[5] F. E. BROWDER and W. V. PETRYSHYN, The solution by iteration of nonlinear functional equations in Banach spaces, Bull. Amer. Math. Soc. 72 (1966), 571-576.

[6] M. BUDZYŃSKA, T. KUCZUMOW and S. REICH, Uniform asymptotic normal structure, the uniform semi-Opial property and fixed points of asymptotically regular uniformly lipschitzian semigroups, Part I, Abstr. Appl. Anal. 3 (1998), 133-151.

[7] E. CASINI and E. MALUTA, Fixed points of uniformly lipschitzian mappings in spaces with uniformly normal structure, Nonlinear Anal. 9 (1995), 103-108.

[8] J. A. CLARKSON, Uniformly convex spaces, Trans. Amer. Math. Soc. 40 (1936), 396-414.

[9] T. DOMÍNGUEZ BENAVIDES, Fixed point theorems for uniformly lipschitzian mappings and asymptotically regular mappings, Nonlinear Anal. 32 (1998), 15-27.

[10] T. DOMÍNGUEZ BENAVIDES and H.-K. XU, A new geometrical coefficient for Banach spaces and its applications in fixed point theory, Nonlinear Anal. 25 (1995), 311-325.

[11] D. J. DOWNING and W. O. RAY, Uniformly lipschitzian semigroups in Hilbert space, Canad. Math. Bull. 25 (1982), 210-213.

[12] D. J. DOWNING and B. TURETT, Some properties of the characteristic of convexity relating to fixed point theory, Pacific J. Math. 104 (1983), 343-350. 
[13] M. EDELSTEIN, The construction of an asymptotic center with a fixed point property, Bull. Amer. Math. Soc. 78 (1972), 206-208.

[14] K. GOEBEL and W. A. KIRK, A fixed point theorem for transformations whose iterates have uniform Lipschitz constant, Studia Math. 47 (1973), 135-140.

[15] K. GOEBEL, W. A. KIRK and R. L. THELE, Uniformly lipschitzian families of transformations in Banach spaces, Canad. J. Math. 26 (1974), 1245-1256

[16] K. GOEBEL and W. A. KIRK, Topics in metric fixed point theory, Cambridge Univ. Press, Cambridge, 1990.

[17] K. GOEBEL and S. REICH, Uniform convexity, hyperbolic geometry, and nonexpansive mappings, Marcel Dekker, New York, 1984.

[18] J. GÓRNICKI, A fixed point theorem for asymptotically regular mappings, Colloq. Math. 64 (1993), 55-57.

[19] J. GÓRNICKI, Fixed points of asymptotically regular mappings in metric spaces, Demonstratio Math. 29 (1996), 615-620.

[20] J. GÓRNICKI, Fixed points of asymptotically regular semigroups in Banach spaces, Rend. Circ. Mat. Palermo 46 (1997), 89-118.

[21] H. ISHIHARA and W. TAKAHASHI, Fixed point theorems for uniformly lipschitzian semigroups in Hilbert spaces, J. Math. Anal. Appl. 127 (1987), 206-210.

[22] T. H. KIM and W. A. KIRK, Fixed point theorems for lipschitzian mappings in Banach spaces, Nonlinear Anal. 26 (1996), 1905-1911.

[23] E. A. LIFSHITZ, Fixed point theorems for operators in strongly convex spaces, Voronez. Gos. Univ. Trudy Mat. Fak. 16 (1975), 23-28.

[24] T. C. LIM, Fixed point theorems for uniformly lipschitzian mappings in $L^{p}$ spaces, Nonlinear Anal. 7 (1983), 555-563.

[25] T. C. LIM, Some $L^{p}$ inequalities and their applications to fixed point theorems of uniformly lipschitzian mappings, Proc. Sympos. Pure Math. 45 (1986), 119-125.

[26] T. C. LIM and H.-K. XU, Uniformly lipschitzian mappings in metric spaces with uniform normal structure, Nonlinear Anal. 25 (1995), 1231-1235.

[27] T. C. LIM, H.-K. XU and Z.-B. XU, An $L^{p}$ inequality and its applications to fixed point theory and approximation theory, Progress in Approximation Theory, (P. Nevai, A. Pinkus, Eds.), Academic Press, (1991), 609-624.

[28] B. PRUS and R. SMARZEWSKI, Strongly unique best approximations and centers in uniformly convex spaces, J. Math. Anal. Appl. 121 (1987), 10-21.

[29] R. SMARZEWSKI, Strongly unique minimization of functionals in Banach spaces with applications to the theory of approximation and fixed points, J. Math. Anal. Appl. 115 (1986), 155-172 .

[30] K.-K. TAN and H.-K. XU, Fixed point theorems for lipschitzian semigroups in Banach spaces, Nonlinear Anal. 20 (1993), 395-404.

[31] D. TINGLEY, Noncontractive uniformly lipschitzian semigroups in Hilbert space, Proc. Amer. Math. Soc. 92 (1984), 355-361.

[32] H.-K. XU, Fixed point theorems for uniformly lipschitzian semigroups in uniformly convex spaces, J. Math. Anal. Appl. 152 (1990), 391-398.

[33] H.-K. XU, Random fixed point theorems for nonlinear uniformly lipschitzian mappings, Nonlinear Anal. 26 (1996), 1301-1311.

[34] L.-C. ZENG, On the existence of fixed points and nonlinear ergodic retractions for lipschitzian semigroups without convexity, Nonlinear Anal. 24 (1995), 1347-1359. 
[35] W. ZHAO, Geometrical coefficients and measures of noncompactness, Ph.D. Dissert., Univ. Glasgow, 1992.

Monika Budzyńska and Tadeusz Kuczumow

INSTYTUT MATEMATYKI UMCS

20-031 Lublin, POLAND

E-mail address: tadek@golem.umcs.lublin.pl

SimEON REICH

Department of Mathematics

The Technion-Israel Institute of Technology

32000 HAIFA, ISRAEL

E-mail address: sreich@techunix.technion.ac.il 


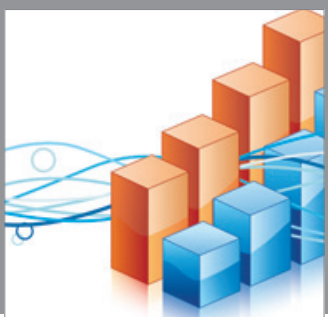

Advances in

Operations Research

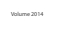

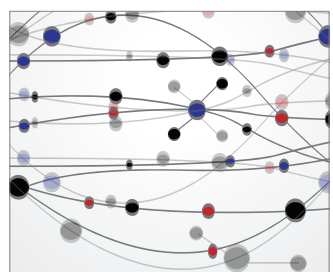

\section{The Scientific} World Journal
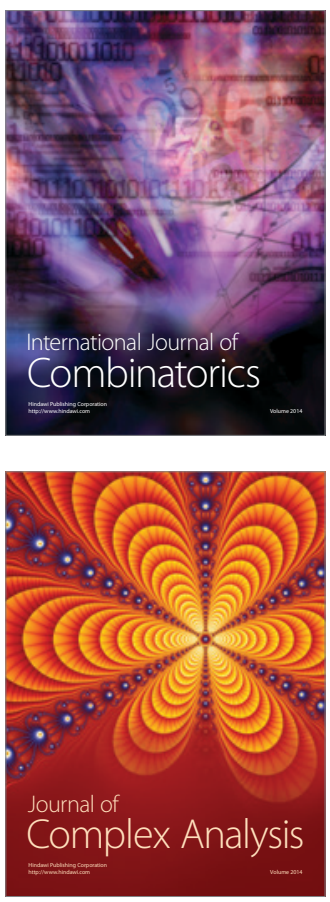

International Journal of

Mathematics and

Mathematical

Sciences
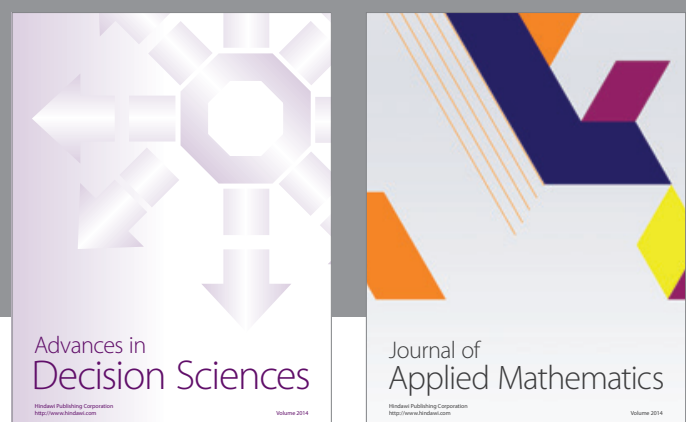

Journal of

Applied Mathematics
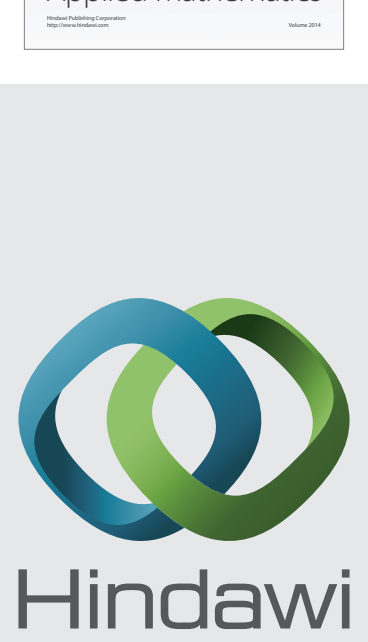

Submit your manuscripts at http://www.hindawi.com
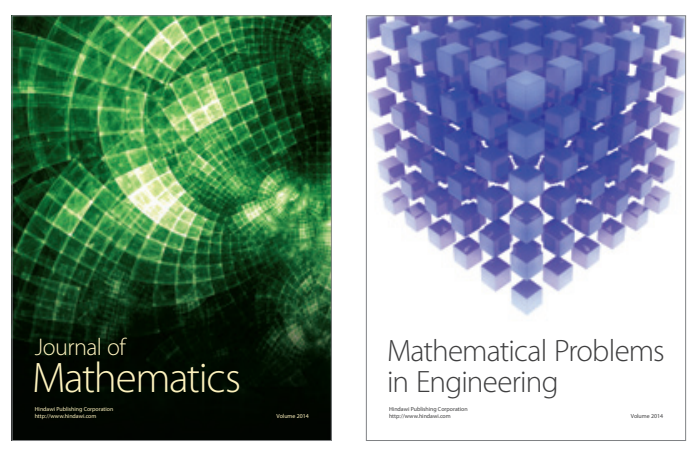

Mathematical Problems in Engineering
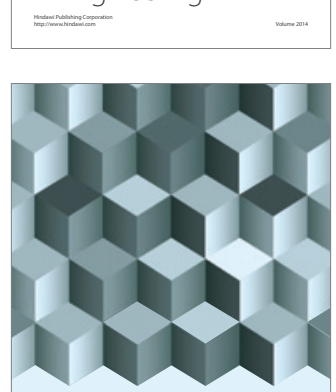

Journal of

Function Spaces
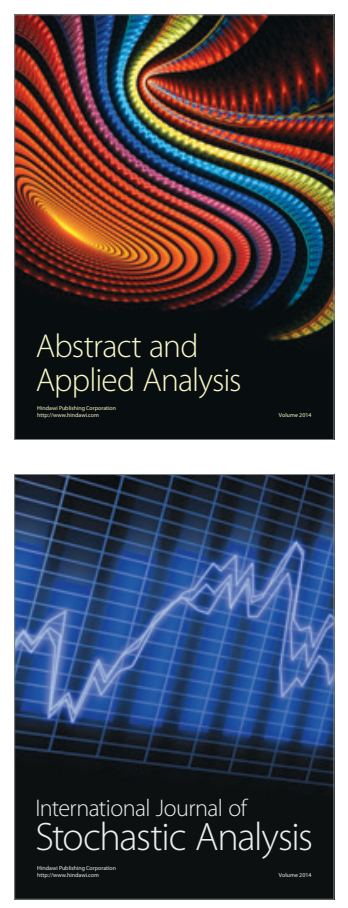

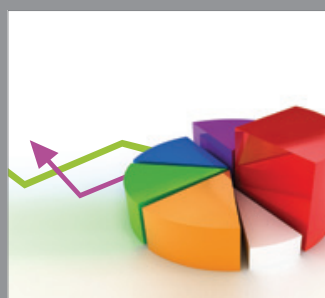

ournal of

Probability and Statistics

Promensencen
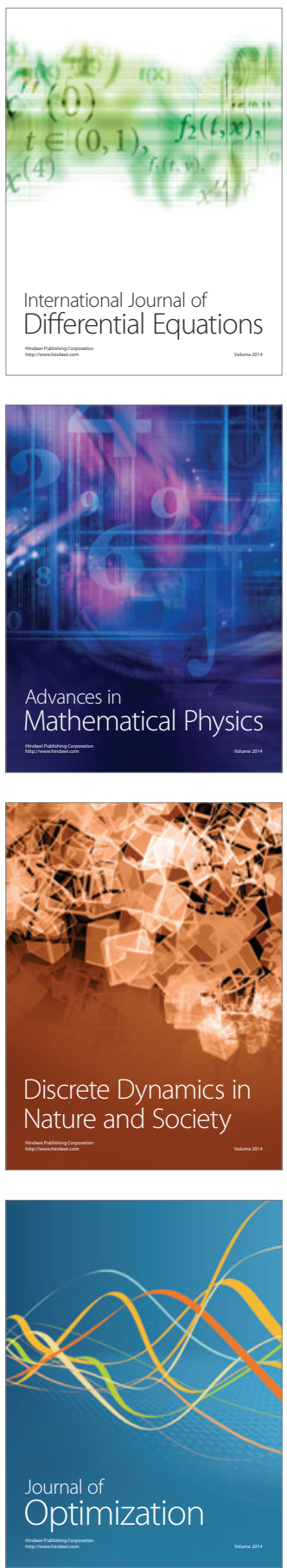